\title{
Critical dynamics near the glass transition as explored with muon spin relaxation
}

\author{
F. J. Bermejo, ${ }^{1,2}$ I. Bustinduy, ${ }^{1,2}$ M. A. González, ${ }^{3}$ S. H. Chong, ${ }^{4}$ C. Cabrillo,${ }^{5}$ and S. F. J. Cox ${ }^{6}$ \\ ${ }^{1}$ C.S.I.C. - Department of Electricity and Electronics, Universidad Basque Country, P.O. Box 644 Bilbao 48080, Spain \\ ${ }^{2}$ Consejo Superior de Investigaciones Científicas, Serrano 123, E-28006 Madrid, Spain \\ ${ }^{3}$ Institut Laue Langevin, Boîte Postale 156x, F-38042 Grenoble Cedex 9, France \\ ${ }^{4}$ Laboratoire de Physique Mathématique et Theorique, Universite Montpellier II, 34095 Montpellier, France \\ ${ }^{5}$ Instituto de Estructura de la Materia, Consejo Superior de Investigaciones Científicas, Serrano 123, E-28006 Madrid, Spain \\ ${ }^{6} I S I S$ Facility, Rutherford Appleton Laboratory, Chilton, Oxon OX11 OQX, United Kingdom and \\ Condensed Matter and Materials Physics Group, University College London, WC1E 6BT, United Kingdom
}

(Received 17 August 2004; published 6 December 2004)

\begin{abstract}
The dynamics of two molecular glass formers are monitored at microsecond scales via muon spin relaxation. Measurements in transverse magnetic fields display unambiguous signatures of critical behavior at temperatures somewhat above those signaling the thermodynamic glass transition, namely at the onset of stochastic molecular motions. The temperature dependence of transverse relaxation rates and amplitudes is found to provide accurate and unbiased estimates for a critical temperature $T_{c}$ as predicted by kinetic theories of the glass transition.
\end{abstract}

DOI: 10.1103/PhysRevB.70.214202

PACS number(s): 67.20.+k, 61.20.Ja, 76.75.+i

\section{INTRODUCTION}

The current view on the microscopic motions within a glassy solid portrays them as mostly vibrational in nature. Some of the most remarkable properties shown by the glassy state are known to arise from groups of atoms which execute large-amplitude motions ${ }^{1}$ having mean-squared displacements larger than the thermal values. This comes as a consequence of the action of anharmonic terms in the interparticle potential ${ }^{2}$ which are manifest in the large thermal expansion coefficients shown by glasses as compared to their parent crystals. Crossing the glass transition temperature $T_{g}$ from below leads to the supercooled liquid, characterized by the onset of diffusive molecular motions which take place in addition to fast vibrations.

The huge viscosity of a deeply supercooled liquid constrains its constituent particles to move by concerted rearrangements with its neighbors, usually involving motions of a different nature (i.e., roto-translational motions, molecular deformational movements, etc.). The onset of such motions occurs when some relevant diffusion coefficient (translational or rotational) attains measurable values and this is then taken as a signature of liquidlike motions resulting from thermal activation of density fluctuations.

To the authors' knowledge, kinetic theories of the modecoupling family (MC), ${ }^{3}$ together with others that make use of the replica idea brought forward from the theory of spin-glasses, ${ }^{4}$ constitute the only formal microscopic tools providing quantitative predictive capabilities about the onset of liquid-flow motions. Schematic versions of MC theories portray the activation of diffusive motions to lie at a characteristic temperature usually referred to as $T_{c}$, located a few tens of Kelvins above $T_{g}$ signaling a transition from an ergodic to a nonergodic phase. Alongside these developments, the theory sketched in Ref. 4 predicts the existence of a critical temperature $T_{f}$ which is below $T_{c}$ at which the con- figurational entropy vanishes and this $\left(T_{f}\right)$ is interpreted as an ideal static glass transition.

Detailed assessments of the soundness of predictions mostly concern results from MC theory. Computer simulation results on Lennard-Jones mixtures lend support to some of the predictions. ${ }^{5}$ In contrast, the experimental scrutiny of such a theory has encountered difficulties resulting from the intricacies of particle motions present in laboratory glass formers. These may obscure the sought dynamical phenomenon. Indeed, efforts to assess the physical soundness of $T_{c}$ require sophisticated data treatments ${ }^{6}$ to unveil hints of critical behavior. This has lead in some cases to disparate estimates for $T_{c}{ }^{7,8}$ since sometimes estimates for $T_{c}$ are close to the lower limit of stability of the normal liquid where particle movements usually reach the picosecond scale. These difficulties arise from the existence in actual materials of motions that are strongly coupled to the density fluctuations and therefore their effect needs to be isolated from the measured response. Such motions may exhibit dependences on temperature quite distinct from that followed by translational mass diffusion and may therefore hide any sign of criticality at $T_{c}$ as it has now been recognized. ${ }^{9}$

Here we report on results on the dynamics of two glass formers [1-propanol (1Pr) and glycerol (Gly)] from low temperatures up to their normal liquid ranges, as explored by implanted positive muons. This follows a preliminary asessment of the capabilities of the technique ${ }^{10}$ to monitor the dynamics of a material upon crossing the liquid $\rightarrow$ glass and crystal $\rightarrow$ liquid transitions. Our aim was to perform a test as stringent as possible on the predictions made from theory using for the purpose two well studied glass formers. In fact, the materials under consideration are small-molecule glass formers and thus amenable to full microscopic calculations ${ }^{16}$ and are well characterized by measurements of their diffusive dynamics by means of quasielastic neutron scattering that explores motions with characteristic times within the nansosecond range. 


\section{EXPERIMENTAL DETAILS}

The measurements were carried out using the EMU spectrometer at ISIS. ${ }^{11}$ The glass samples were prepared by a deep quench into liquid nitrogen, prior to insertion into a cryostat kept well below $T_{g}(98 \mathrm{~K}$ for $1 \mathrm{Pr}$ and $185 \mathrm{~K}$ for Gly). Spectra of the glassy samples under longitudinal (LF) and transverse fields (TF) were then measured from $5 \mathrm{~K}$ up to temperatures corresponding to the normal liquid range (200-300 K, respectively, corresponding to melting temperatures of 148 and $285 \mathrm{~K}$, respectively).

The measurements reported in this paper are closely analogous to those of $T_{1}$ (LF or spin-lattice relaxation) and $T_{2}$ (TF or spin-spin relaxation), familiar in conventional magnetic resonance. They are known collectively as $\mu \mathrm{SR}$ (muon spin relaxation or rotation), an acronym clearly contrived to resemble ESR (electron spin resonance). In fact, our $\mu \mathrm{SR}$ studies combine analogies with both ESR and NMR (nuclear magnetic resonance), since the implanted positive muons can reach both paramagnetic and electronically diamagnetic states. These elementary particles mimic the chemical behavior of protons, either picking up an electron to form muonium $\left(\mathrm{Mu}=\mu^{+} e^{-}\right)$- the analog of atomic hydrogen (we refer to this as the paramagnetic fraction)-or else thermalizing in diamagnetic states. In alcohols, ROH, this latter is undoubtedly the positive ion $\mathrm{ROHMu}^{+}$in the first instance, formed in the manner of direct protonation, but subsequent proton exchange can lead to effective substitution in the neutral molecule, thus ROMu. Details concerning the energetics and geometry of such diamagnetic fraction were investigated by means of $a b$ initio Hartree-Fock calculations on isolated molecules. In both cases, the stablest molecular configuration corresponds to a muon stuck to the hydroxyl oxygen at a distance of $0.966 \AA$. The protonated species $\mathrm{ROHMu}^{+}$displays a geometry where the bound muon, $\mathrm{OH}$ group, and the adjacent carbon lie in a plane so that the closest distance between $\mathrm{Mu}$ and closest hydrogen lies at $1.64 \AA$.

Our TF $\mu$ SR measurements display only the diamagnetic fraction, i.e., either $\mathrm{ROHMu}^{+}$or $\mathrm{ROMu}$ (according to temperature, as we see below). The inclusion of a small $\mathrm{HMu}$ fraction, formed by an abstraction reaction of atomic muonium, is also not excluded. The TF signals are thus analogous to free induction decays in proton NMR and correspond to precession of the muon spin at or near its Larmor frequency. (The much higher precession signals corresponding to atomic muonium are invisible at a pulsed muon source such as ISIS, at the magnetic fields used.) The LF signal, on the other hand, subsumes both paramagnetic and diamagnetic fractions: one expects to find exponential spin-lattice relaxation of the muonium fraction towards a baseline set by the diamagnetic fraction. One must bear in mind that thermal-equilibrium polarization is negligible compared with the initial muon polarization on implantation (which is close to $100 \%$ ) but that mechanisms of longitudinal relaxation on the microsecond time scale of these measurements (set by the muon lifetime, $\tau_{\mu}=2.2 \mu \mathrm{s}$ ) exist only for the paramagnetic fraction. ${ }^{12,13}$

\section{RESULTS}

Measurements carried out under longitudinal fields yield exponential relaxation signals having decay rates, ampli-
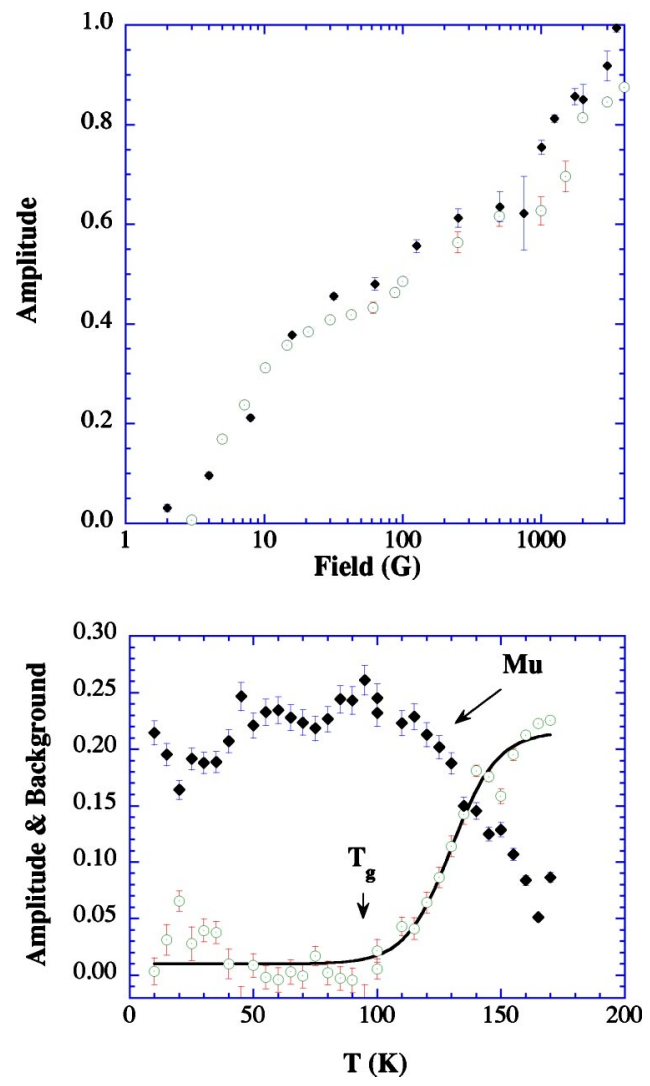

FIG. 1. The upper frame shows the LF dependence of signal fractional amplitude as measured at $100 \mathrm{~K}$ for $1 \mathrm{Pr}$ (open symbols) and Glycerol (closed symbols). The lower frame depicts the temperature dependence of the relaxation amplitude (closed symbols) and the temperature-dependent background term for 1Pr measured under a LF field of $100 \mathrm{G}$. The solid line depicts a fit to a function described below.

tudes, and baseline values that are significantly dependent upon temperature and applied field.

The examples provided in Fig. 1 show how the LF relaxation amplitude varies with temperature and applied magnetic field. The latter decouples the muon spin successively from the local fields generated by nearby proton nuclear moments (typically a few tens of Gauss) and then from the muonium electron (the hyperfine field in atomic muonium is $1600 \mathrm{G}$ ). The resultant two-step increase in the relaxing signal amplitude is seen in the upper frame of Fig. 1. The muonium fraction, thus measured in fields at or above $\approx 100 \mathrm{G}$, proves to depend on temperature. This variation is displayed in the lower frame of Fig. 1 which shows how the relaxing amplitude decreases above $T_{g}$ at the expense of a constant background term attributable to diamagnetic species. This finding suggests that the $\mathrm{ROHMu}^{+}$ion is a center for muonium formation via capture of radiolytic electrons. The muonium yield is then a function of the ionic lifetime and the electron arrival time. The electron must arrive and be captured while the center is still positively charged. That appears to be the case below $100 \mathrm{~K}$ in 1 Pr and below $200 \mathrm{~K}$ in Gly. For comparison, the corresponding temperature in $\mathrm{H}_{2} \mathrm{O}$ ice is around $150 \mathrm{~K}$, where the increase in diamagnetic fraction has been shown to correspond to its change from an ionic to 


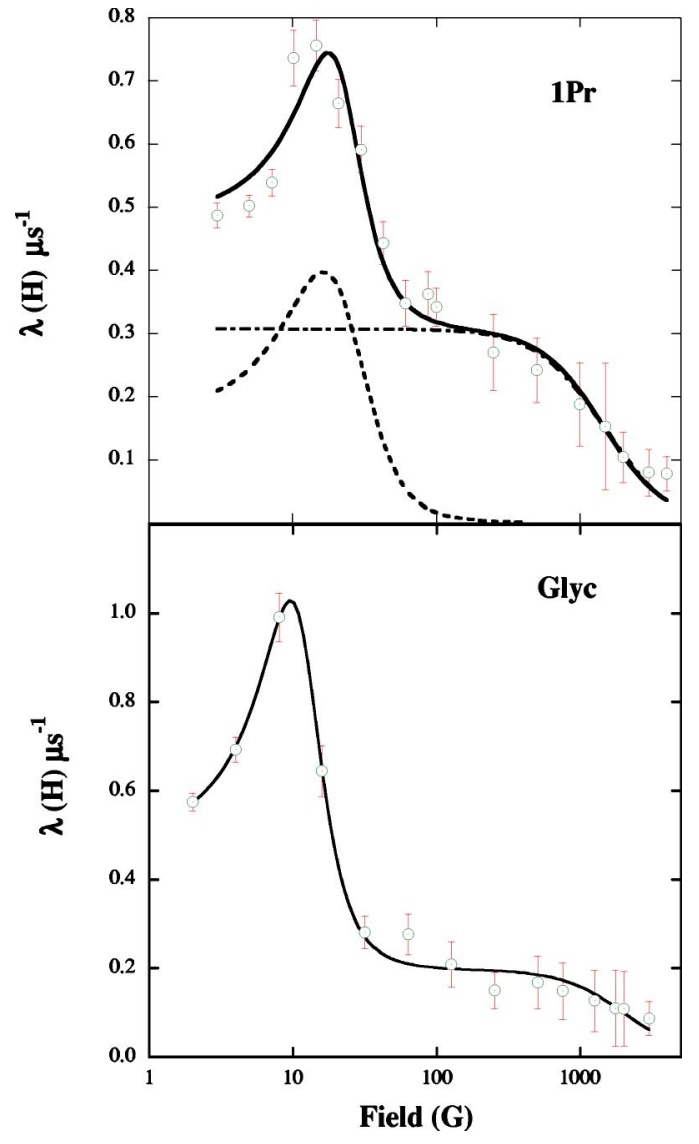

FIG. 2. The upper frame shows the LF dependence of the relaxation rate as measured at $T=100 \mathrm{~K}$ for $1 \operatorname{Pr}$ (symbols), fitted with resonant and nonresonant Lorentzians. The lower frame shows data for Glycerol for $T=100 \mathrm{~K}$.

a neutral species by means of ${ }^{17} \mathrm{O}$ quadrupole resonance. ${ }^{14,15}$ Above $200 \mathrm{~K}$ in $\mathrm{Pr}$ and above $300 \mathrm{~K}$ in Gly the proton loss rate (i.e., the $\mathrm{ROHMu}^{+}$to $\mathrm{ROMu}$ conversion rate) must be faster than the electron capture rate, suppressing that route to muonium formation and leaving only the prompt or epithermal yield.

The field dependence of the longitudinal relaxation rates at a given temperature for the two samples is shown in Fig. 2. The two datasets correspond to a sample within the deeply supercooled liquid close to the glass transition (1Pr) as well to another within the arrested (glass-) phase (Glyc). For both cases, the variation of relaxation rate with magnetic field can be described by a superposition of two Lorentzians, the one broad and centered on zero field, the other a narrower resonance centered at a small nonzero field. (A similar twocomponent variation is noticeable in data for other $\mathrm{OH}-$ containing materials. ${ }^{10}$ )

The narrow component is centered at about $18 \mathrm{G}$ for $1 \mathrm{Pr}$ and about $10 \mathrm{G}$ for Glyc, with a linewidth of 13 and $7 \mathrm{G}$, respectively. Its resonant nature suggests an anticrossing of energy levels for the muonium fraction, associated either with proton superhyperfine interactions or some small anisotropic component of the muon-electron hyperfine coupling. Of importance to the present dynamical study is the underlying broad Lorentzian, which essentially maps the spectral density function or power spectrum for muonium diffusion. Suppression of the relaxation in the vicinity of the muonium hyperfine field, namely $1600 \mathrm{G}\left(2 \pi A / \gamma_{e}\right.$ with $\left.A=4.5 \mathrm{GHz}\right)$ is indicative of fast diffusion, namely $\tau \leqslant 1 /(2 \pi A) \sim 35$ ps. Whereas the muonium diffusion rate is undoubtedly a function of the local dynamics of the host molecules, the two are not simply related. We make no further use of the longitudinal-field data but note that, in situations where muonium would react with the host molecules (e.g., when these are unsaturated organics), the resulting spin label would provide a very direct measure of relevant correlation times, extending the dynamic range to shorter times by two orders of magnitude (i.e., by the ratio of electron and muon magnetic moments). In the following, we confine our attention to the transverse-field data for the diamagnetic fraction, i.e., for the interplay of $\mathrm{ROHMu}^{+}$and $\mathrm{ROMu}$.

Measurements performed in TF are sensitive to static or "frozen-in" spatial variations of the local magnetic field since these induce a dephasing of the muon spin precession. At low temperatures, strong damping of the cosine precession signals results from the vector sum of internal plus applied fields, so that individual muons precess at slightly different Larmor frequencies. The spectra yield relaxation or damping rates that are the simple inverse of the $T_{2}$ relaxation times: $\lambda_{T F}(T)=T_{2}^{-1}(T)$. These relaxation rates are expected to decrease with increasing temperature due to the onset of molecular motions: this is analogous to motional narrowing in NMR, though there can also be a reduction in the static linewidth on going from the ionic to the substituted species $\left(\mathrm{ROHMu}^{+} \rightarrow \mathrm{ROHMu}\right)$. In contrast, the corresponding amplitude of the precession signal is expected to increase as muonium formation is suppressed, so that the diamagnetic muon fraction increases. An example of the experimental spectra for $1 \mathrm{Pr}$ at two temperatures is shown in Fig. 3.

The results for the two glasses are shown in Figs. 4 and 5. In both cases, a mild decrease in relaxation rate is accompanied by a small increase in signal amplitudes up to the calorimetric glass-transition temperatures. There are no clear features at $T_{g}$ but a strong decrease in relaxation rates, accompanied by a marked increase in signal amplitude, is clearly seen at higher temperatures. Finally a clear change of regime is attained for temperatures some 1.3 times $T_{g}$ where the relaxation rates follow a smooth, approximately linear, dependence with increasing temperature. No data were taken above $200 \mathrm{~K}$ (1Pr) or $305 \mathrm{~K}$ (Gly) since more rapid motions (faster than $10^{-8} \mathrm{~s}$ ) go beyond the dynamic range of our instrument.

Figures 4 and 5 show that molecular motions within the glass phases (i.e., $T<T_{g}$ depart from thermally activated behavior as expected for a harmonic solid [i.e., $\ln \lambda(T) \propto 1 / T$ ]. The temperature dependence of such relaxation rates mimics that shown by the average mean-squared displacement. ${ }^{2}$ This suggests that part of the relaxation may be attributed to intermolecular dipolar interactions. On the basis of such an analogy we model the temperature dependence of the relaxation rates well below $T_{g}$ making allowance for higher power terms in the temperature dependence of the mean-squared displacement. Such behavior arises because of the anharmonic terms in the potential and explicit formulas to model them in detail are given in Ref. 2. The resulting rates are 


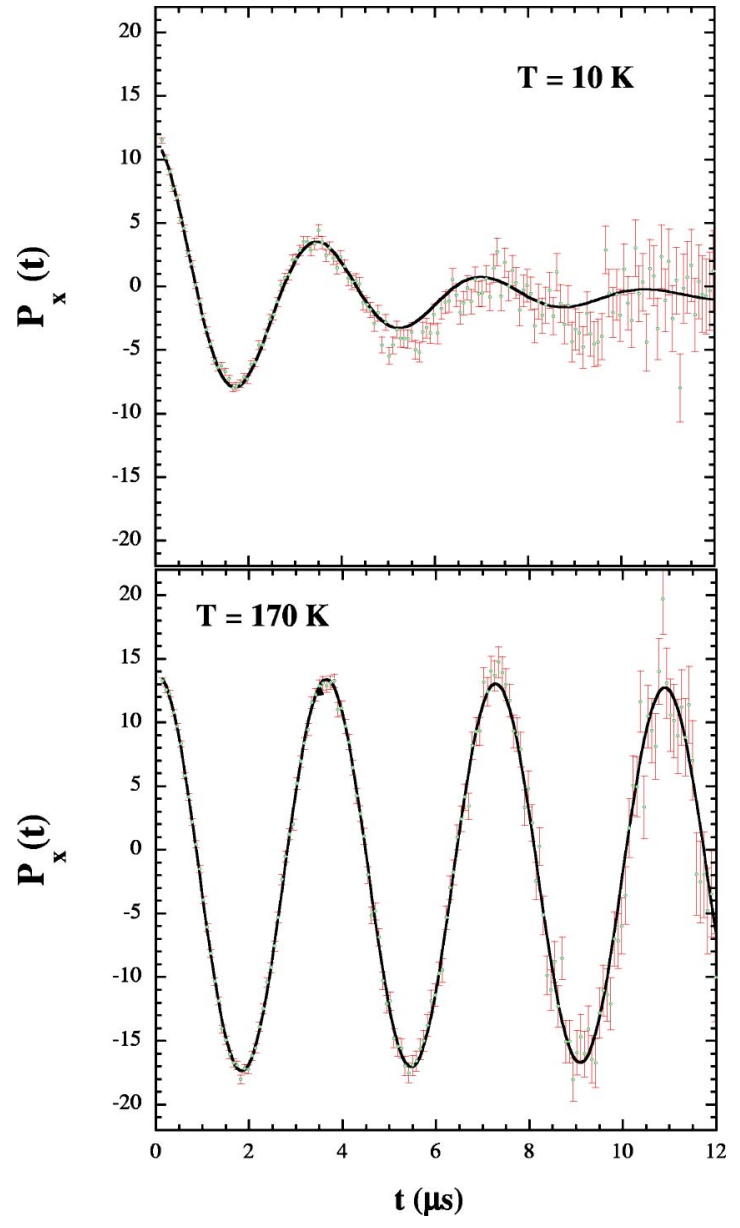

FIG. 3. Transverse field spectra for $1 \mathrm{Pr}$ at low and high temperatures (symbols). The solid line is a fit to a damped cosine function. Notice the near recovery of a pure cosine signal at the higher temperature.

describable up to $0.5 T_{g}$ by $\lambda_{\text {glass }}(T)=A \exp \left(a T+b T^{2}\right)$, with temperature coefficients of $-3.01 \times 10^{-4} \mathrm{~K}^{-1}$ and 0.0 for $1 \mathrm{Pr}$ and $-6.27 \times 10^{-3} \mathrm{~K}^{-1}$ and $-1.31 \times 10^{-5} \mathrm{~K}^{-2}$ for glycerol, respectively. Values for $\lambda_{\text {glass }}(T \rightarrow 0)$ close to those found by extrapolation of experimental data were also calculated from particle configurations derived from molecular simulations. ${ }^{17}$

Above $T_{g}$ the relaxation rates shown in Figs. 4 and 5 exhibit a strong drop that cannot be attributed to the same motions causing its mild decrease with temperature referred to above. The shape of such curves is reminiscent of that predicted by mode-coupling theories (MCT) $)^{3,16}$ of the glass transition concerning the Lamb-Mössbauer factor. In fact, our data can be described if a term $\propto \sqrt{\left|T-T_{c}\right| / T_{c}}$, where $T_{c}$ stands for a critical temperature, is included. In other words, the temperature dependence of the relaxation rate provides clear evidence of criticality appearing about a temperature $T_{c}$ and the data from the deep-glass phase up to the normal liquid can be accounted for by

$$
\begin{gathered}
\lambda(T)=A \lambda_{\text {glass }}(T) \sqrt{T_{\text {red }}}+\zeta\left(T_{\text {red }}\right) ; \quad T \leqslant T_{c} \\
\lambda(T)=\zeta\left(T_{\text {red }}\right) ; \quad T>T_{c}
\end{gathered}
$$

with

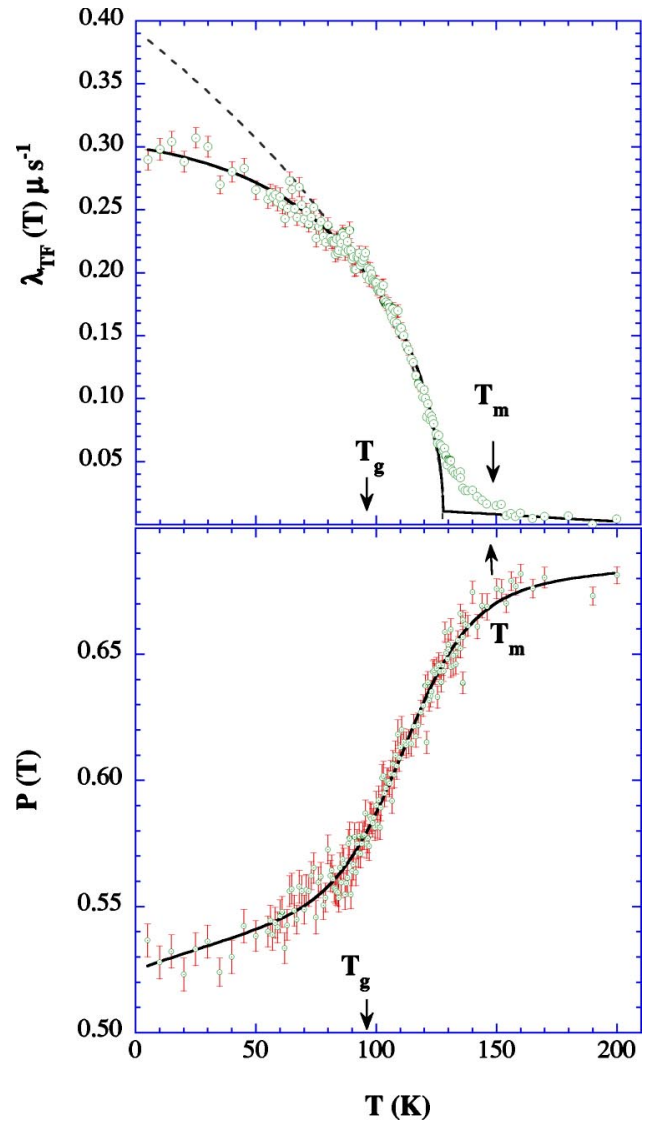

FIG. 4. Experimental TF relaxation rates normalized to the value extrapolated for zero temperature $\lambda_{\text {glass }}(T \rightarrow 0)$ (symbols, upper frame) and normalized relaxation amplitudes (symbols, lower frame) for 1-propanol as well as the approximations described in the text (solid lines). Arrows with $T_{g}$ and $T_{m}$ signal the glass transition and crystal melting temperatures, respectively. The dashed line shows the prediction made by MCT (see below).

$$
T_{\text {red }}=\left|T-T_{c}\right| / T_{c}, \quad \zeta\left(T_{\text {red }}\right)=\alpha_{0}+\beta_{0} T_{\text {red }} .
$$

The high temperature data are in both cases modeled by a mild, linear temperature dependence with coefficient values easily calculated from the measured data once the value for $T_{c}$ is set.

The data shown in Figs. 4 and 5 for the normalized signal amplitudes $P(T)$ provide additional information concerning the dynamics within the supercooled liquid range. For both glasses one sees, superimposed on a gradient, a sigmoidshaped curve with onset temperatures close to $T_{g}$ and saturation values somewhat below $T_{m}$. Their shapes are reminiscent of plots of the quantity $1-f$ where $f$ stands for the nonergodicity parameter reported by a number of studies ${ }^{8}$ and attributed to the fraction of excitations that decay by fast processes. Lacking a theoretical recipe to account for such data we have empirically modeled it as resulting from a growth process involving the number of particles taking part in diffusive motions. It follows a generalized logistic function

$$
P(T)=L a+U a /\left\{1+\exp \left[-r\left(T-T_{c}\right)\right]\right\}
$$

where parameters $L a$ and $U a$ stand for the lower and upper asymptotes and can be determined directly from the data, $T_{c}$ 


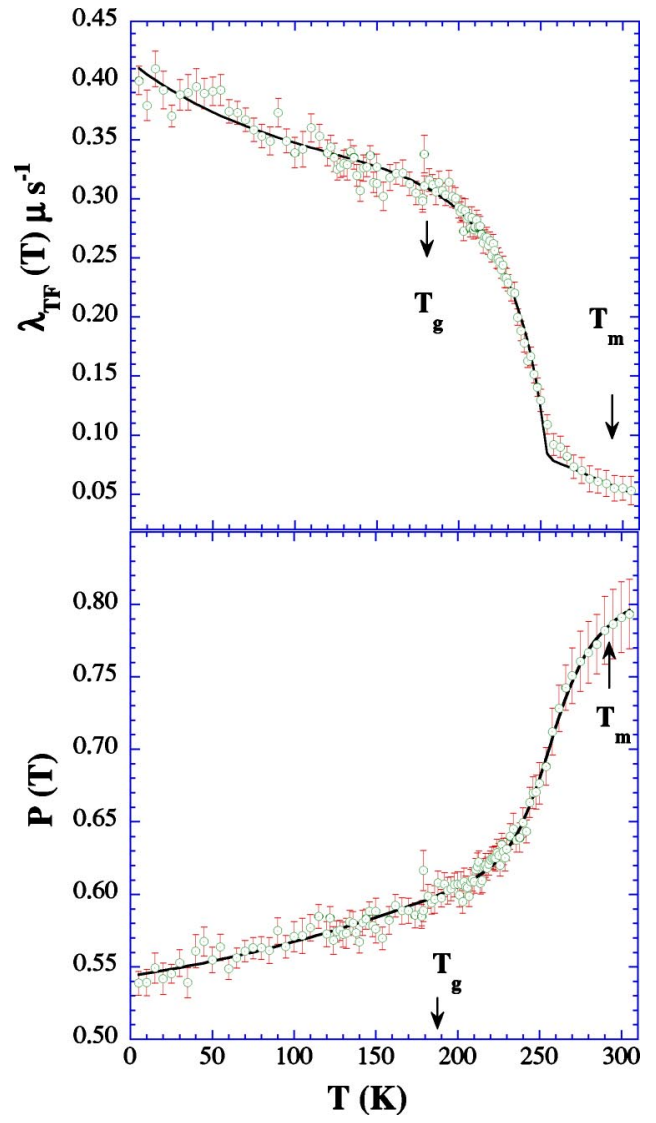

FIG. 5. Experimental TF relaxation rates (symbols, upper frame) and relaxation amplitudes (symbols, lower frame) for glycerol as well as the approximations described in the text (solid lines). The rest of symbols retain the same meaning as in Fig. 4.

is here identified with the temperature where maximum growth occurs and $r$ stands for the growth rate. Fitting both the relaxation rate and amplitude curves using $T_{c}$ and $r$ as adjustable parameters yields values of $T_{c}=129(2) \mathrm{K}$ for $1 \mathrm{Pr}$ and 259 (4) for Gly, together with values of $0.027(2) \mathrm{K}^{-1}$ and $0.087(7) \mathrm{K}^{-1}$ for the respective growth rates. The benefit of making use of the signal amplitudes as well as the relaxation rates is an overall reduction in the error-limits and consequently a more accurate estimate of this elusive temperature. Notice that Eq. (3) also accounts for the increase in amplitude associated with diamagnetic species concomitant with a decreasing fraction of atomic muonium as shown in Fig. 1.

\section{DISCUSSION AND CONCLUSIONS}

The significance of the present results is now assessed by consideration of the reported values for $T_{c}$ for materials such as glycerol. ${ }^{7}$ Estimates for such a temperature range from $225 \mathrm{~K}$ (i.e., $1.2 T_{g}$ ) up to $310 \mathrm{~K}\left(19 \mathrm{~K}\right.$ above $\left.T_{m}\right)$, depending not only on the technique employed but also upon the analysis routes followed in each case. Data reported in Ref. 8 for $\alpha$-picoline also predict a crossover temperature far too close to that signaling melting. The physical soundness of our estimates for $T_{c}$ for both samples here reported on is best proven by independent observations of neutron quasielastic broadenings which arise as a result of Doppler broadening of an elastic line. These provide clear indications of the activation of diffusive motions that take place with frequencies comparable or faster than a few GHz. For the two glasses studied here, such motions take place at temperatures rather close to our predicted $T_{c}$ 's, i.e., at about $120 \mathrm{~K}$ for $1 \operatorname{Pr}^{18}$ and $250 \mathrm{~K}$ for Gly. ${ }^{7,19}$ This provides direct evidence of the existence of a somewhat smeared-out singularity at some temperature above the calorimetric glass transition as predicted by $\mathrm{MC}$ theories of the glass transition. The results also serve to rule out estimates for such a temperature for one of our samples ${ }^{7}$ since, at temperatures where $T_{c}$ is predicted to be located, stochastic motions within the picosecond scale have been reported. ${ }^{7,19}$

A comparison of our present results for glycerol to Mössbauer data ${ }^{20}$ lends additional support to our interpretation. Mössbauer absorption spectra of dissolved ${ }^{57} \mathrm{Fe}^{2+}$ show significant broadening above $T=250 \mathrm{~K}$ from which relaxation times of about $30 \mathrm{~ns}$ and mean-square displacements of about $0.02 \AA^{2}$ are reported. The data show a strong temperature dependendence leading to values of about $1 \mathrm{~ns}$ and $0.035 \AA^{2}$ at $T=270 \mathrm{~K}$. Since the iron probe is not expected to stick to molecules, the motions felt by it are identified with those giving rise to the $\alpha$-relaxation peak manifest in dielectric relaxation experiments. The values for the relaxation time and particle mean-squared displacement derived from this experimental probe can be compared to those derived from neutron quasielastic scattering and therefore provide yet another independent test concerning the region of temperatures where stochastic motions set in. A further point worth noticing concerns recent data ${ }^{21}$ showing that anhydrous glycerol crystallizes at about $263 \mathrm{~K}$, a temperature that also signals a strong drop in correlation between molecular dipoles.

The large values reported recently as estimates for $T_{c}$ mostly resulting from scaling analysis of dielectric spectroscopy data ${ }^{7}$ may point towards some dynamic phenomenon that develops at temperatures close to melting of the stable crystal. An indication of such a change of regime above some temperature $T \approx 290 \mathrm{~K}$ is given by macroscopic viscosity data plotted as $\eta^{-1 / 2}$ as suggested by Taborek et al. ${ }^{22}$ and shown in Fig. 6.

Figure 6 shows that at high temperatures and in common with many other glass-forming materials, the viscosity follows a power-law behavior. Extrapolation to lower temperatures of such a behavior yields an intercept at $T=290 \mathrm{~K}$ that comes rather close to the recent estimate of $T_{c}=288 \pm 3$ reported by Adichtchev et al. ${ }^{7}$ from a scaling analysis of dielectric spectroscopy data. Since at temperatures well above $T_{g}$ one expects the dielectric relaxation frequencies for the main $(\alpha-)$ peak to closely follow the temperature dependence of the shear viscosity, the results from the analysis just referred to seem to reflect the change in behavior experienced by the viscosity rather than the onset of diffusive motions.

To explore the predictive capability of the MC theory as developed for molecular materials, an explicit calculation was carried out for propanol. The relevant details of such a calculation are given in Ref. 23. Intermolecular interactions 


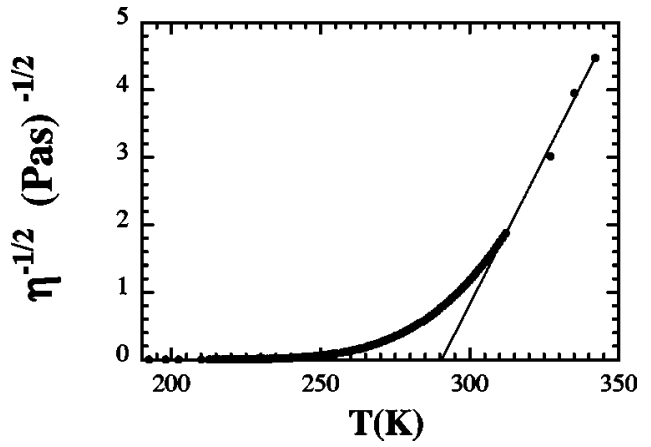

FIG. 6. Temperature dependence of the viscosity represented as suggested in Ref. 22. Closed symbols are experimental points and the solid line represents an extrapolation of the high-temperature, normal-liquid behavior.

are represented in terms of a site-site description of the molecular correlations involving five force-centers per molecule. The required input was a set of partial static structure factors $S_{i j}(Q)$ derived from computer molecular dynamics simulations using five interaction sites. The model potential here employed has been thoroughly tested against experimental data, ${ }^{24,25}$ and found to reproduce the most salient features of the static (pair distribution functions) and dynamic (frequency spectrum) correlations of this material. The estimate given for $T_{c}=325 \mathrm{~K}$ is obviously far too high $\left(\approx 3.3 T_{g}\right)$. However, setting its value to that found by experiment and using the calculated value for the exponent parameter $\gamma=0.686$ one calculates the temperature dependence of the anomaly in the relaxation rate by means of the expression for the square-root singularity quoted above. The result is also shown in Fig. 4. There one sees that the prediction accounts for the shape of our relaxation data for temperatures ranging from $T_{g}$ up to $T_{c}$.

The results here reported illustrate the advantages of $\mu \mathrm{SR}$ for these kinds of studies. The technique is especially suited for studies on single-particle dynamics within the mesoscopic range where predictions made by microscopic theories are expected to be most reliable. Because of the time window accessible to $\mu \mathrm{SR}$, only relatively low-frequency motions (up to $\mathrm{MHz}$ scales) contribute to the relaxation rate. This allows the sought phenomenon - that is the onset of molecular stochastic motions - to stand out from other motions strongly coupled to it that may dominate the response measured by higher-frequency probes such as neutron and light scattering. As a consequence, the technique reveals the presence of somewhat smeared out critical behavior taking place at temperatures a few tens of degrees above $T_{g}$ thus providing a truly unbiased confirmation of the theoretical prediction.

\section{ACKNOWLEDGMENTS}

Work supported in part by Grant No. MAT2002-04540C05-03 (Spain). The authors are grateful to the ISIS staff for technical assistance.
${ }^{1}$ D. Caprion and H. R. Schober, Phys. Rev. B 62, 3709 (2000); R. Fernández-Perea, F. J. Bermejo, and E. Enciso, ibid. 53, 6215 (1996); K. Vollmar-Lee, W. Kob, K. Binder, and A. Zippelius, J. Chem. Phys. 116, 5158 (2002).

${ }^{2}$ C. Cabrillo, M. A. Gonzalez, G. J. Cuello, F. J. Bermejo, M. L. Saboungi, and D. L. Price, Phys. Rev. B 69, 134202 (2004).

${ }^{3}$ W. Götze, J. Phys.: Condens. Matter 11, A1 (1999).

${ }^{4}$ M. Mezard and G. Parisi, Phys. Rev. Lett. 82, 747 (1999).

${ }^{5}$ W. Kob and H. C. Andersen Phys. Rev. E 51, 4626 (1995); 52, 4134 (1995).

${ }^{6}$ H. Z. Cummins, J. Phys.: Condens. Matter 11 A95 (1999); H. C. Barshilia, G. Li, G. Q. Shen, and H. Z. Cummins, Phys. Rev. E 59, 5625 (1999).

${ }^{7}$ E. Rössler, A. P. Sokolov, A. Kisliuk, and D. Quitmann, Phys. Rev. B 49, 14967 (1994); J. Wuttke, J. Hernandez, C. Li, G. Coddens, H. Z. Cummins, F. Fujara, W. Petry, and H. Sillescu, Phys. Rev. Lett. 72, 3052 (1994); S. Adichtchev, T. Blochowicz, C. Tschirwitz, V. N. Novikov, and E. A. Rössler, Phys. Rev. E 68, 011504 (2003); P. Lunkenheimer, A. Pimenov, M. Dressel, Yu. G. Goncharov, R. Böhmer and A. Loidl, Phys. Rev. Lett. 77, 318 (1996); T. Franosch, W. Götze, M. R. Mayr, and A. P. Singh, Phys. Rev. E 55, 3183 (1997); D. M. Paolucci and K. A. Nelson, J. Chem. Phys. 112, 6725 (2000).

${ }^{8}$ S. V. Adichtchev, St. Benkhof, Th. Blochowicz, V. N. Novikov, E. Rössler, Ch. Tschirwitz, and J. Wiedersich, Phys. Rev. Lett. 88, 055703 (2002).
${ }^{9}$ R. Schilling, J. Phys.: Condens. Matter 12, 6311 (2000).

${ }^{10}$ C. Cabrillo, F. J. Bermejo, and S. F. J. Cox, Phys. Rev. B 67, 184201 (2003).

${ }^{11} \mathrm{http}: / / \mathrm{www}$. isis.rl.ac.uk/muons/emu

${ }^{12}$ S. F. J. Cox, Solid State Nucl. Magn. Reson. 11, 103 (1998).

${ }^{13}$ For a general review of $\mu$ SR spectroscopy see, for instance, S. F. J. Cox, J. Phys. C 20, 3187 (1987).

${ }^{14}$ S. F. J. Cox, J. A. S. Smith, and M. C. R. Symons, Hyperfine Interact. 65, 993 (1990).

${ }^{15}$ S. F. J. Cox, Phys. Scr., T 45, 292 (1992).

${ }^{16}$ T. Theenhaus, R. Schilling, A. Latz, and M. Letz, Phys. Rev. E 64, 051505 (2001); A. Winkler, A. Latz, R. Schilling, and C. Theis, ibid. 62, 8004 (2000); M. Letz, R. Schilling, and A. Latz, ibid. 62, 5173 (2000); R. Schilling, ibid. 65, 051206 (2002).

${ }^{17}$ The quantities are related to the second moment of the field distribution generated by proton nuclear moments with a magnetogyric ratio $\gamma_{H}$ on the muon site and are given by $\Delta M$ $=\frac{1}{5} \Sigma_{i}\left[\mu_{0} / 4 \pi / \hbar \gamma_{\mu} \gamma_{H} / d_{\mu-i}^{3}\right]^{2}$ where $\gamma_{\mu}$ is the muon magnetogyric ratio and $d_{\mu-i}$ are distances between proton nuclei and the muon bound to the alcohol molecule. The location of the latter has been determined by ab initio molecular orbital calculations. The value calculated for $1 \mathrm{Pr}$ yields $0.32 \mu \mathrm{s}^{-1}$, to be compared with $0.30 \mu \mathrm{s}^{-1}$ determined by extrapolation of experimental data.

${ }^{18}$ F. J. Bermejo, W. S. Howells, M. Jimenez-Ruiz, M. A. Gonzalez, D. L. Price, M. L. Saboungi, and C. Cabrillo, Phys. Rev. B 69, 174201 (2004). 
${ }^{19}$ D. J. Tomlinson, Mol. Phys. 25, 735 (1972); G. J. Cuello, F. J. Bermejo, R. Fayos, R. Fernandez-Perea, A. Criado, F. Trouw, C. Tam, H. Schober, E. Enciso, and N. G. Almarza, Phys. Rev. B 57, 8254 (1998); see also F. J. Bermejo, A. Criado, A de Andres, E. Enciso, and H. Schober, ibid. 53, 5259 (1996).

${ }^{20}$ G. U. Nienhaus, H. Fraunfelder, and F. Parak, Phys. Rev. B 43, 3345 (1991).

${ }^{21}$ Ya. E. Ryabov, Y. Hayashi, A. Gutina, and Y. Feldman, Phys. Rev. B 67, 132202 (2003).

${ }^{22}$ P. Taborek, R. N. Kleiman, and D. J. Bishop, Phys. Rev. B 34, 1835 (1986).

${ }^{23}$ S. H. Chong and W. Götze, Phys. Rev. E 65, 041503 (2002).
${ }^{24}$ C. Talón, F. J. Bermejo, C. Cabrillo, G. J. Cuello, M. A. Gonzalez, J. W. Richardson, Jr., A. Criado, M. A. Ramos, S. Vieira, F. L. Cumbrera, and L. M. Gonzalez, Phys. Rev. Lett. 88, 115506 (2002).

${ }^{25}$ C. Talón, G. J. Cuello, M. A. González, F. J. Bermejo, C. Cabrillo, and R. Connatser, Chem. Phys. 292, 263 (2003); M. A. González, E. Enciso, F. J. Bermejo, and M. Bee, Phys. Rev. B 61, 6654 (2000); M. A. González, E. Enciso, F. J. Bermejo, M. Jimenez-Ruiz, and M. Bee, Phys. Rev. E 61, 3884 (2000); M. A. González, F. J. Bermejo, E. Enciso and C. Cabrillo, Philos. Mag. 84, 1599 (2004). 